\title{
An Automatic Classification Algorithm of Digital Image Based on Semantics
}

\author{
Ling Xing ${ }^{*}$, Wei Zhao and Rong Fu
}

\author{
School of Information Engineering, Southwest University of Science and Technology, Mianyang, Sichuan 621010, China
}

\begin{abstract}
The randomness and fuzziness of digital image semantics are hot issues in the field of classification technology, so a new semantic representation of digital image based on cloud model is proposed and a semantic vector space is constructed. In the space, semantic classifications of digital images are completed by calculating the semantic class certainty degree (SCCD). In addition, we propose cloud support vector machine based on image semantics (CSVM-IS) model, which can effectively utilize the knowledge of SCCD. At the same time, CSVM-IS can automatically and effectively classify the multi-semantic information and eliminate the rejection of the classification samples. We design two kinds of simulation experiments to verify the classification performance. One experiment is to verify the classification accuracy, and the other is to verify the classification effectiveness. Experimental results show that CSVM-IS is superior to the Nesting Algorithm in terms of classification performance.
\end{abstract}

Keywords: Cloud Model, Semantic Class Certainty Degree, Semantic Characteristic.

\section{INTRODUCTION}

With the development of network technology and the growing number of users, the massive fragmented digital image resources increase the time and cost consumption to be obtained valid information. At present, the early image classification methods are based on color, texture and shape of low-level features or the combined features with the lowlevel features. Generally, low-level features were extracted and analyzed, and the obtained feature space was represented as matrices $[1,2]$. Then the images were classified by calculating the characteristic value of the matrices $[3,4]$. With the constant increasing of users' requirements for image retrieval, the semantic indexing technology came into being. The image segmentation was proposed and the classification was completed with the symbiotic probability of the segmentation areas and key words [5]. And the emotional semantic classification method was put forward [6]. In the image classification methods, SVM-based classification was widely used for its good performance [7, 8]. These classifiers worked well for a single semantic processing, but for multisemantics, the rejected classification was relatively serious, so the classification effect was poor. Although fuzzy theories could improve the original classification of digital image, they cannot completely express the correlation among digital image semantics [9].

In view of the problems about semantic gap and weak semantic correlation, digital image classification is implemented by calculating the semantic class certainty degree (SCCD) and then the calculated values should form the ex

*Address correspondence to this author at the School of Information Engineering, Southwest university of Science and Technology, Mianyang, Sichuan 621010, China; E-mail: xingling_my@163.com perience sample knowledge to effectively simulate the classification learning models. In this paper, we propose a new classification method, named cloud support vector machine based on image semantics (CSVM-IS), to automatically complete the image classification with the high-level multisemantic information.

\section{CLOUD MODEL}

Membership Clouds Generator (MCG) is proposed to express the uncertain transforming relationship between qualitative concepts and their quantitative description [10]. Cloud generators can fully express the randomness and fuzziness of objective things, and it can also transform the accurate data into the qualitative language values $(\mathrm{Ex}, \mathrm{En}, \mathrm{He})$. Cloud model is one of the mapping relationship model between the qualitative analysis and quantitative knowledge. Cloud generator is generation algorithm of cloud model, and includes forward cloud generator and backward cloud generator.

Definition 1: The certainty degree is the subjection degree that an element $\mathrm{x}$ belongs to the concept $\mathrm{T}$, and it can be expressed in $C_{T}(x), C_{T}(x) \in[0,1]$.

Definition 2: $\mathrm{U}$ is a quantitative set of precise values, $X \subseteq U$. T is a qualitative concept of $\mathrm{U}$, if element $\mathrm{x}(x \in X)$ is a random number with stable tendency for certainty degree, cloud is mapping distribution of $\mathrm{T}$ from $\mathrm{U}$ to $[0,1]$ in data space [11].

Definition 3: Cloud drop is a binary combined with element $\mathrm{x}$ and its certainty degree $C_{T}(x)$, and it can be expressed in $\operatorname{Drop}\left(x, C_{T}(x)\right)$. 


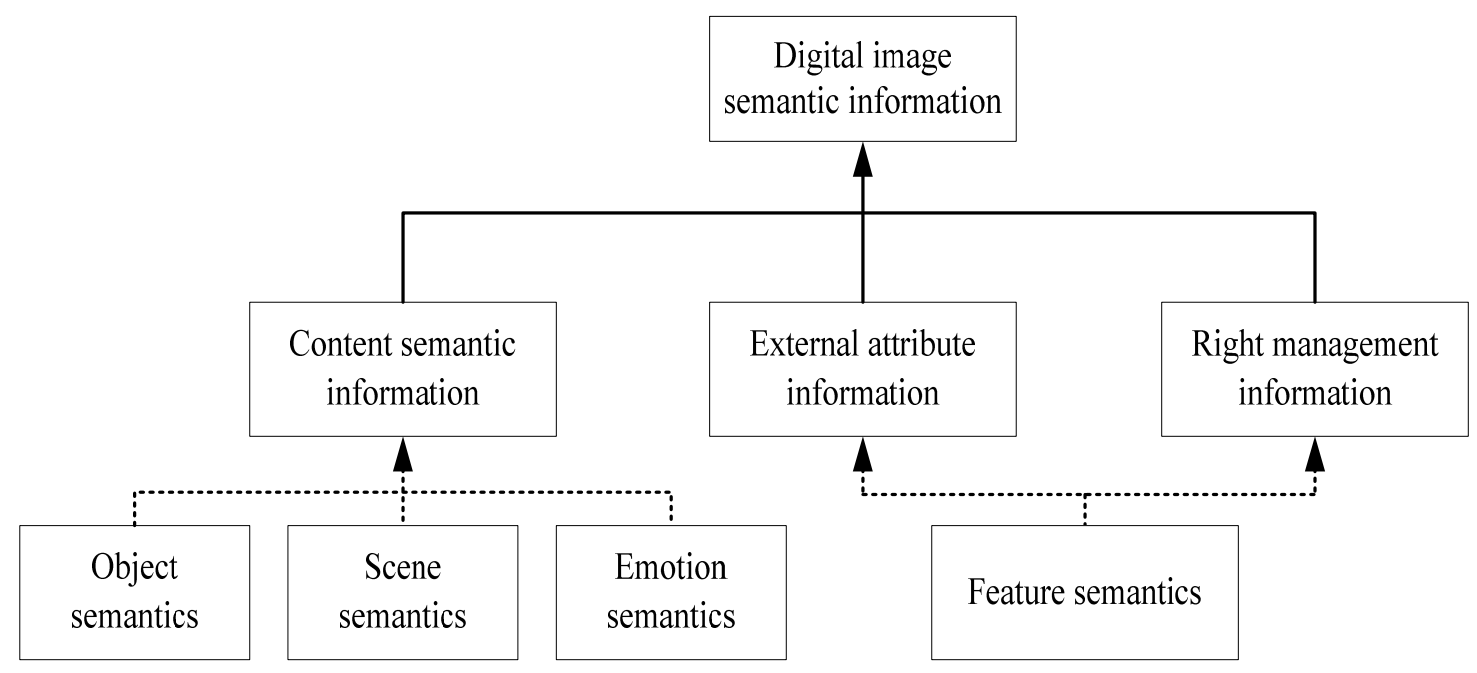

Fig. (1). The semantic level model.

The digital characteristics of cloud model would reflect the qualitative concept of the quantitative characteristic, and there are three parameters to express the characteristics: Ex (expectation), En (entropy) and He (hyper entropy):

Ex (expectation) is the value of the qualitative concept in data space, which can reflect the cloud gravity center of all cloud drops.

En (entropy) is the random measurement of the qualitative concept, and it reflects the dispersion of cloud drops. At the same time, En is also the ambiguity of qualitative concept, and it reflects the value range of cloud drops accepted by the qualitative concept in data space. Therefore, En can reveal the relevance characteristic of the fuzziness and the randomness.

(3) He (hyper entropy) is the entropy of En, and it reflects the condensation degree of cloud drops.

\section{IMAGE SEMANTIC CLASSIFICATION AND LEA- RNING MODEL}

\subsection{Features Expression of Digital Image}

Any semantics of each digital image can be expressed a vector in digital image semantic space. Uniform Content Locator of Image (UCL-I) makes multi-dimensional indexing for categories, themes, sources and authors of digital image $[12,13]$. Through calculating the UCL-I vector, it is easy to precisely locate the image resources by content in the information space.

UCL-I vector can be expressed in Eq. (1):

$U=\left(U_{1}, U_{2}, U_{3}\right)=\left(\left(u_{1}, u_{2}, u_{3}, u_{4}\right),\left(u_{5}, u_{6}, u_{7}\right),\left(u_{8}, u_{9}, u_{10}\right)\right)$

Digital image semantic information is divided into content semantic information, right management information, and external attribute information as shown in Fig. (1). Content semantic information U1 includes theme, title and description information. Right management information U2 includes creator, provider and publish date. External attribute information U3 includes file size, image size and compressed format, and so on.

\subsection{Classification Algorithm of SCCD Based Cloud Model}

Digital image semantic set in UCL-I vector space is U, and $U=\left(u_{1}, u_{2}, \cdots, u_{k}, \cdots, u_{N}\right), u_{k}$ is the k-th semantic information content. All of the digital image semantic informations have $\mathrm{C}$ categories, $u_{i k}$ belongs to category $i, i=1,2, \cdots, C$, and $u_{i k}$ is the k-th semantic component, which can be expressed in Eq.2:

$u_{k j}=\left(u_{k 1}, u_{k 2}, \cdots, u_{k m}\right)$

Some calculation formulas are given as follows.

Average value of semantic component:

$\bar{U}_{k j}=\frac{1}{m} \sum_{j=1}^{m} U_{k j}$

Variance of semantic component:

$S_{k j}^{2}=\frac{1}{m-1} \sum_{j=1}^{m}\left(U_{k j}-\bar{U}_{k j}\right)^{2}$

Expectation of semantic component:

$E_{x k j}=\bar{U}_{k j}$

Entropy of semantics:

$E_{n k j}=\sqrt{\frac{\pi}{2}} \times \frac{1}{m} \times \sum_{j=1}^{m}\left|U_{k j}-E_{x k j}\right|$

Hyper entropy of semantics:

$H_{e k j}=\sqrt{S_{k j}^{2}-E_{n k j}^{2}}$

Semantic radius: 


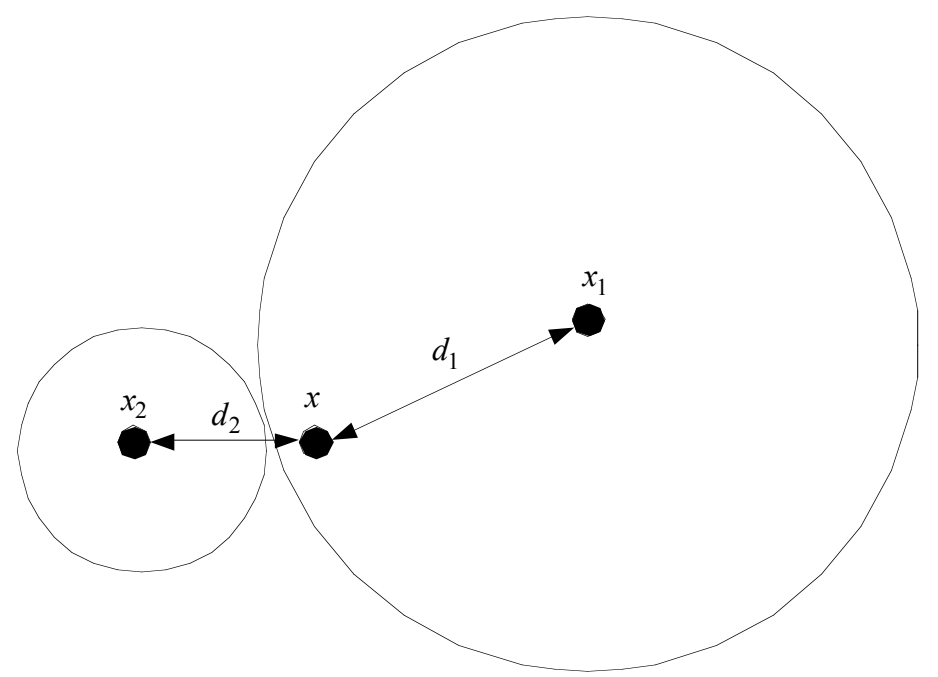

Fig. (2). The classification based on the membership degree of distance.

$R_{k j}=\max \left|U_{k j}-P_{k j}\right|(j=1,2, \cdots, m)$

Certainty degree of samples $U_{k}$ :

$C_{T}(k)=1-\left\|\frac{U_{k}-P_{k}}{R_{k}}\right\| \quad(k=1,2, \cdots, N)$

Through calculating the above semantic eigenvalues, images that have the same semantics are gathered at great extents, at the same time, images which have different semantics are separated. The closer distance between samples and semantic focus are, the bigger certainty degree is, and vice versa.

In existing classification methods, the membership degree of classification samples is usually composed of semantic distance, expectation and entropy between classifycation samples and classification semantics. This method ignores the randomness and fuzziness of semantic barycenter, so it will bring with the classification errors. The situation of classification errors is shown in Fig. (2).

In Fig. (2), two circles represent two categories, $\mathrm{x} 1$ and $\mathrm{x} 2$ are respectively the category centers, and $\mathrm{x}$ is the awaiting classification sample. At the same time, $\mathrm{d} 1$ and $\mathrm{d} 2$ are the distance between $\mathrm{x}$ to $\mathrm{x} 1$ and $\mathrm{x} 2, d_{1}>d_{2}$. Although $\mathrm{x}$ belongs to the large circle, it is classified to the small circle because of shorted distance to the small one. The classification error is caused by the distance deviation. In order to solve the classification error, the cloud membership degree is put forward and the definition is shown as Eq. (10).

Definition 4: The semantic cloud membership degree is the membership degree of awaiting sample to a certain of training sample. It can be expressed by the probability of the category, so the semantic cloud membership S(k)of awaiting semantic sample $u$ to the training sample $\mathrm{k}$ can be calculated with Eq. 10 .

$S(k)=1-\left\|\exp \left(-\frac{2 \operatorname{En}(k)^{2}}{u-E_{x}(k)^{2} * H_{e}(k)}\right)\right\|$ $(k=1,2, \cdots, N)$

In Eq. (10), $\mathrm{k}$ is the number of training sample categories, and $E_{x}(k), E n(k), H_{e}(k)$ respectively represent the k-class expectation, entropy and hyper entropy. Then, the semantic cloud membership degree of rejected sample can be calculated with Eq.11.

$\mathrm{u}_{k} \in w_{C}, C=\arg \left\{\max _{\mathrm{k}}(S(\mathrm{k}))\right\}$

In Eq. (11), $u_{k}$ is rejected sample, $w_{C}$ is the C-class of training sample, and $S(k)$ is the semantic cloud membership degree of $u_{k}$ to the training sample $\mathrm{k}$.

According to the Eq. (11), the bigger entropy of semantic class is, the bigger the semantic cloud membership degree is, then the bigger the probability of the category which awaiting semantic sample belong to is. The bigger hyper entropy is, the smaller the semantic cloud membership degree is, the less conducive to the determination of awaiting semantic sample. So, the semantic cloud membership degree, is proportional to the entropy of training category, is inversely proportional to hyper entropy of training category.

Cloud model increases hyper entropy in qualitative description of the concept on the basis of entropy. Therefore this method can fully describe the uncertainties of the objective things. Through the analysis of users' retrieval habits, it is found that using single semantic component in retrieval is unable to meet the needs of users. Generally speaking, the greater the number of semantics, the less of rejected sample, the more efficient the classification becomes. Therefore, in this paper, the multi-semantics classification is adopted, and five semantic characteristics are selected, including topic, title, description, creator and provider.

\section{CSVM-IS MODEL}

Because of the semantic correlation of digital images, Cloud support vector machine based on image semantics (CSVM-IS) is proposed. The structure diagram of CSVM-IS is shown in Fig. (3). The certainty degree can be obtained by 


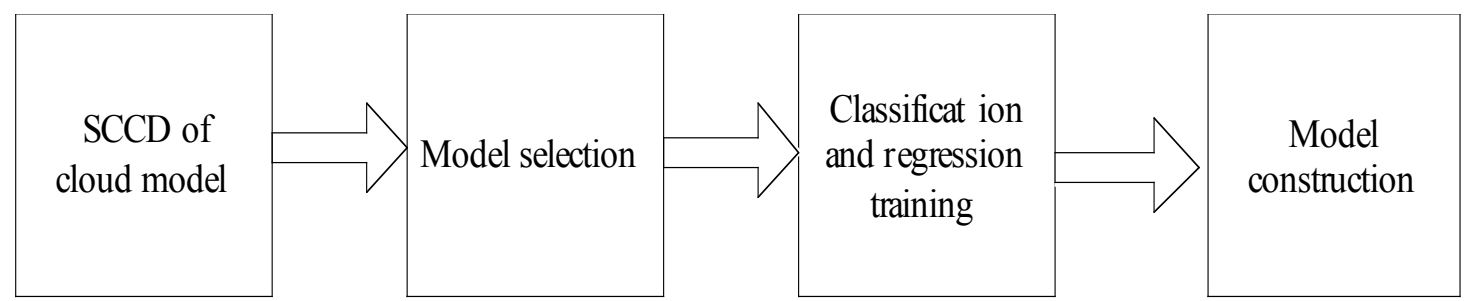

Fig. (3). CSVM-IS classification model.

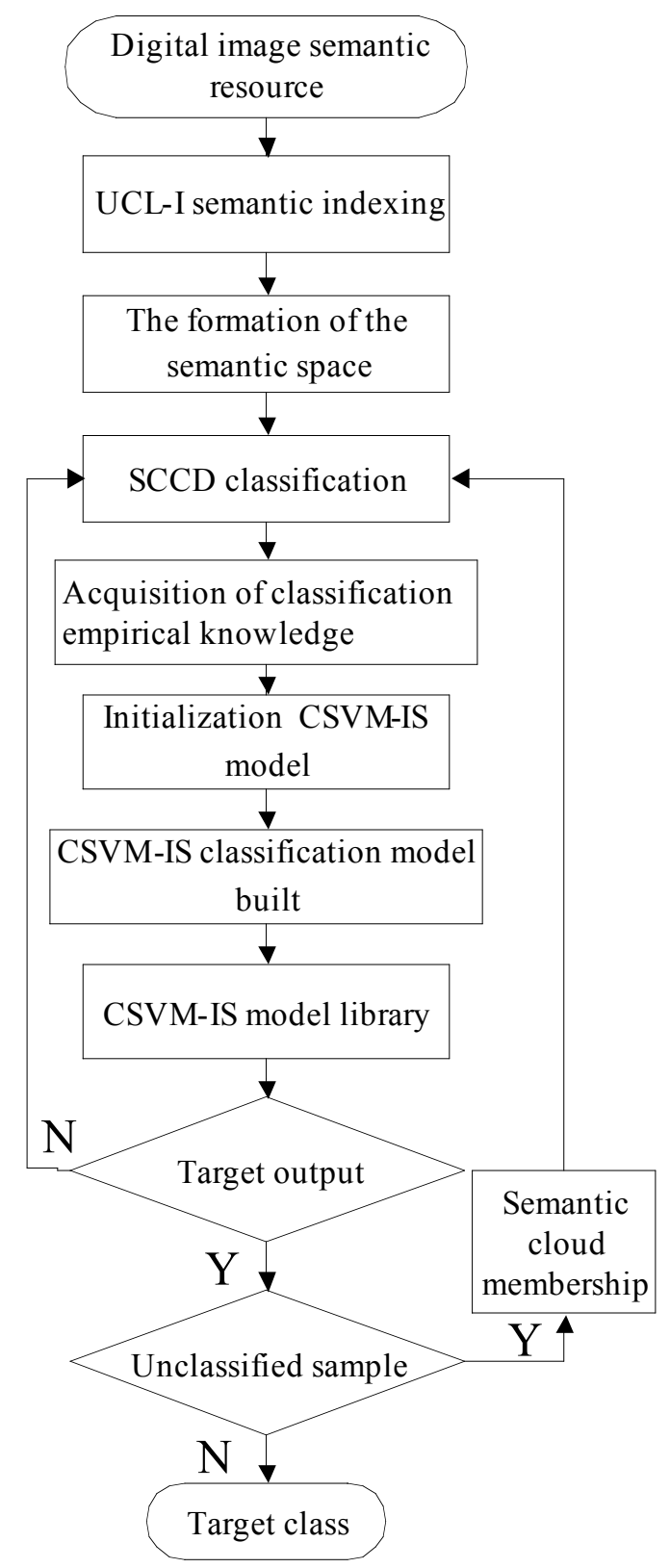

Fig. (4). CSVM-IS classification system flow chart.

the experience knowledge of semantic classification information based on cloud model. Model selection module is to determine the best parameter values with optimization algorithm. Classification and regression training module will train the classification and regression model to simulate semantic classification. Model construction module will complete the automatic classification of image objects based on CSVM-IS model. And the flow chart is shown in Fig. (4).
(1) The UCL-I semantic indexing information is represented by vector.

(2) Semantic radius $P_{k j}$ and SCCD of all of the training samples are calculated, and then data experience knowledge will be obtained which is the classification result of SCCD. 
(3) CSVM-IS model will be initialized, and the sample set is divided into the training set and the test set.

(4) The sample data will be processed using the studying model, and CSVM-IS model is established.

(5) The automatic semantic classification of digital images is completed using CSVM-IS model. If it can find the classification target, then continue to judge whether the sample is the rejected sample. If it isn't the rejected sample, then it is the target class. And if it is the rejected sample, then calculate the inverse cloud membership of each training category $\mathrm{S}(\mathrm{i}),(\mathrm{i}=1,2, \ldots, \mathrm{m})$ and return to the SCCD classification to re-classify.

\section{EXPERIMENTS AND DISCUSSION}

We indexed respectively the eight categories of image resources with UCL-I, and the eight categories included people, animals, plants, nature, architecture, items, scenes and creation as numbering $1,2,3,4,5,6,7$ and 8 . Then $16000 \mathrm{im}$ ages of Caltech datasets [14] are selected to index and get 10 UCL-I vectors which five semantic characteristics, topic, title, description, creator and provider, are selected to be simulated and analyzed. In simulation, the five semantic characteristics are divided into four kinds of data combinations (DC) as shown: DC1 (theme, description), DC2 (title, creator), DC3 (description, provider), DC4 (theme, creator). Through cloud model, the characteristic data of each image including expectation, entropy and hyper entropy would be obtained. We collected the 2000 groups characteristic data of each type image information as the training and testing samples of CSVM-IS classification model.

In this paper, it mainly simulates and analyzes two aspects as follows, the one is to classify the eight categories of image resources; the other is to classify character images based on whether the character images are the target class; then typical Nesting Algorithm in reference [15] is compared with to prove the effectiveness of the algorithm in this paper. The two types of classification are introduced in section 5.1 and 5.2.

\subsection{Semantic Multi-classification Simulation}

In view of the indexing image sample information, $60 \%$ of them are randomly selected as training sample set and $40 \%$ as testing sample set. Comparison between the experience true value and classification predicted values can reflect the effect of semantic classification based on CSVM-IS. And the validity of the proposed method will embody by comparing with the Nesting Algorithm (NA) [11]. In order to facilitate the demonstration, 7 indexing images are stochastically selected from each kind of images, and 20 simulation results for each group data samples are taken the average values. The simulations are shown in Fig. (5).

In Fig. (5), “*” indicates the original sample, "o" indicates the classified forecasting result with CSVM-IS, and " $\square$ " indicates the classified forecasting result with NA. Among them, (a) is the classification performance simulation for DC1, (b) is the one for DC2, (c) is the one for DC3 and (d) is the one for DC4. The upper half of each figure is the classification performance simulation for training sample set and the bottom half is the classification performance simulation for testing sample set. The classification results of two classification algorithms mainly reflect the coincidence between the predicted classification value and true value of sample category. If they are coincident, the classification is correct, otherwise it is false. In the top half of (a), for 56 training samples, the wrong classification number is 3 with CSVM-IS, and is 31 with NA. And in the below one of (a), for 56 testing samples, the wrong classification number is 5 with CSVM-IS. In the top half of (b), for 56 training samples, the wrong classification number is 4 with CSVM-IS, and is 30with NA. And in the below one of (b), for 56 testing samples, the wrong classification number is 5 with CSVMIS, and is 27 with NA. So, the classification error rate is less than $10 \%$ with CSVM-IS, but the error rate is about $50 \%$ with NA. Likewise, in the top half of (c), for 56 training samples, the wrong classification number is 4 with CSVMIS, and is 32 with NA. And in the below one of (c), for 56 testing samples, the wrong classification number is 4 with CSVM-IS, and is 28 with NA. So, the classification error rate is less than $8 \%$ with CSVM-IS, but the error rate is at least $50 \%$ with NA. In the top half of (d), for 56 training samples, the wrong classification number is 5 with CSVMIS, and is 35 with NA. And in the below one of (d), for 56 testing samples, the wrong classification number is 8 with CSVM-IS, and is 26 with NA. So, the classification error rate is about $10 \%$ with CSVM-IS, but the error rate is about $50 \%$ with NA. The above comparison show that CSVM-IS has higher classification accuracy in dealing with multisemantic classification information.

\subsection{Classification Simulation of Semantic Target Class}

To further verify the effectiveness of the algorithm, simulation is analyzed by considering users' demands on image resource classification and whether images belong to the target class. This paper focuses on considering the character image resources, therefore, the character image class serves as the target class and others are non-target class. Simulations of target classification aim at four multi-semantic groups (DC1, DC2, DC3 and DC4), and 60 image sample resources are randomly selected to be analyzed. According to cloud model algorithm, exception, entropy and hyper entropy of eigenvalue are mapped as one number as shown in Eq. (12).

$$
F=E_{x k j}-10 E_{n k j}+10 H_{e k j}
$$

According Eq.12, corresponding data is mapped to the vicinity of the value of 0 , and the semantic resources corresponding eigenvalue of each sample can be obtained, its classification simulation results are shown in Fig. (6).

In Fig. (6), "+" indicates the target class (the image of the character class); “*” indicates non-target category. Closed curve is used as a classification line to distinguish the target class. The image semantic samples in the circle are the target class resource, otherwise there are the non-target class. In (a), the horizontal axis represents the theme, and the vertical 

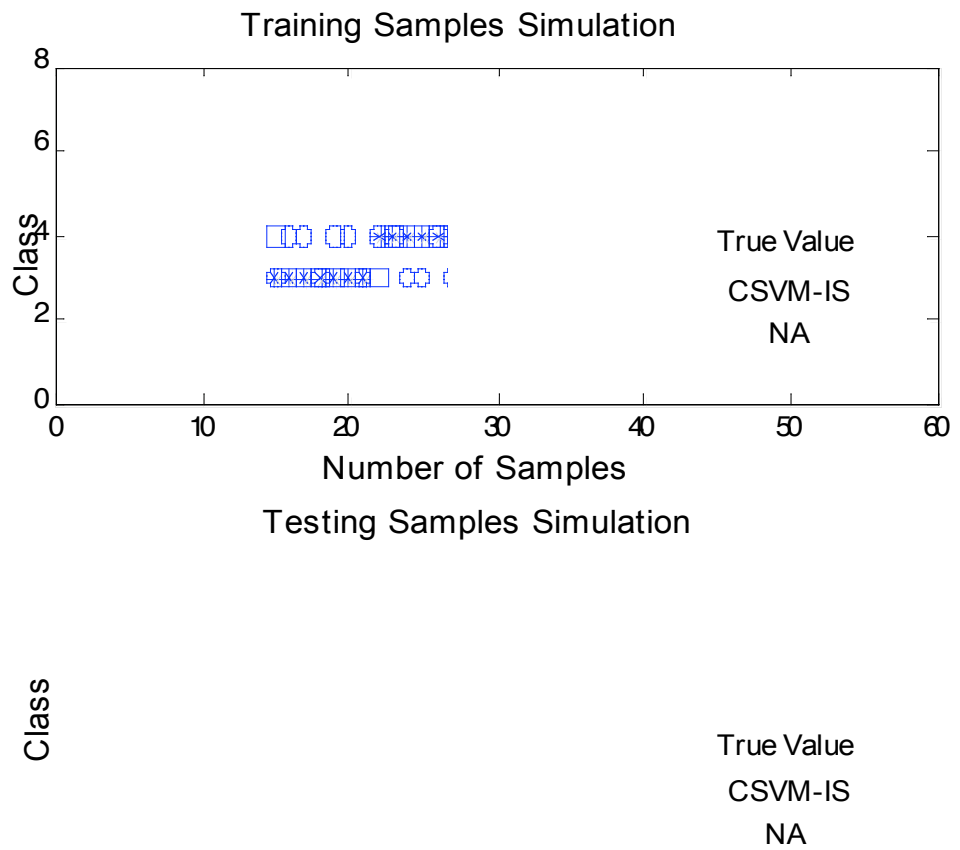

Number of Samples

(a)

Training Samples Simulation

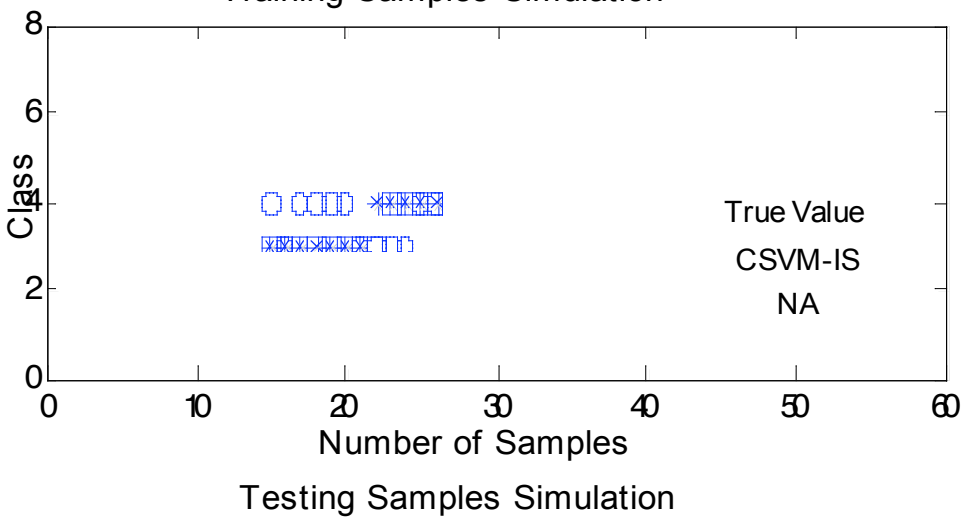

$\frac{\mathscr{W}}{\mathscr{0}}$

True Value

CSVM-IS

NA

Number of Samples

(b) 


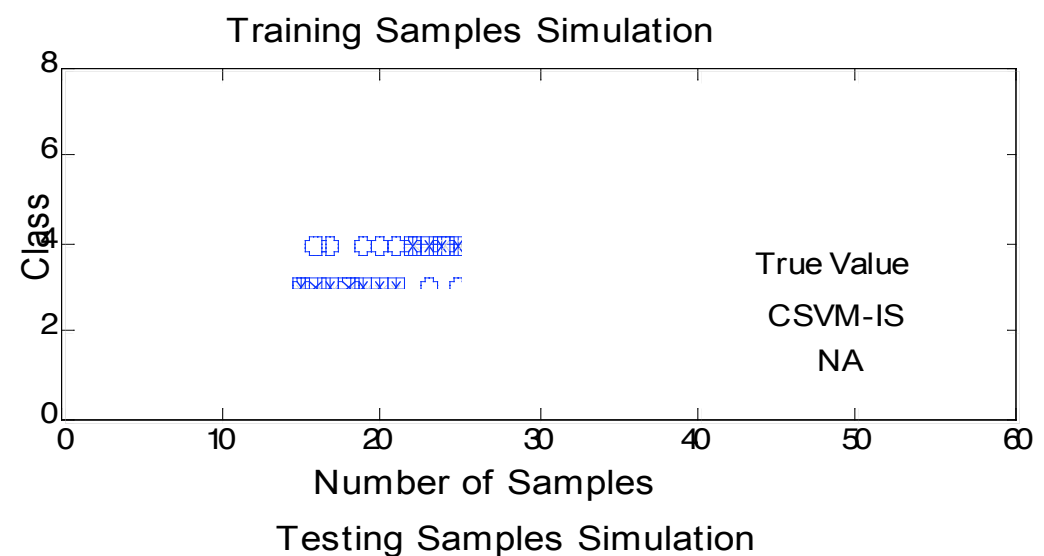

0
0
$\frac{0}{0}$

True Value

CSVM-IS

NA

Number of Samples

(c)
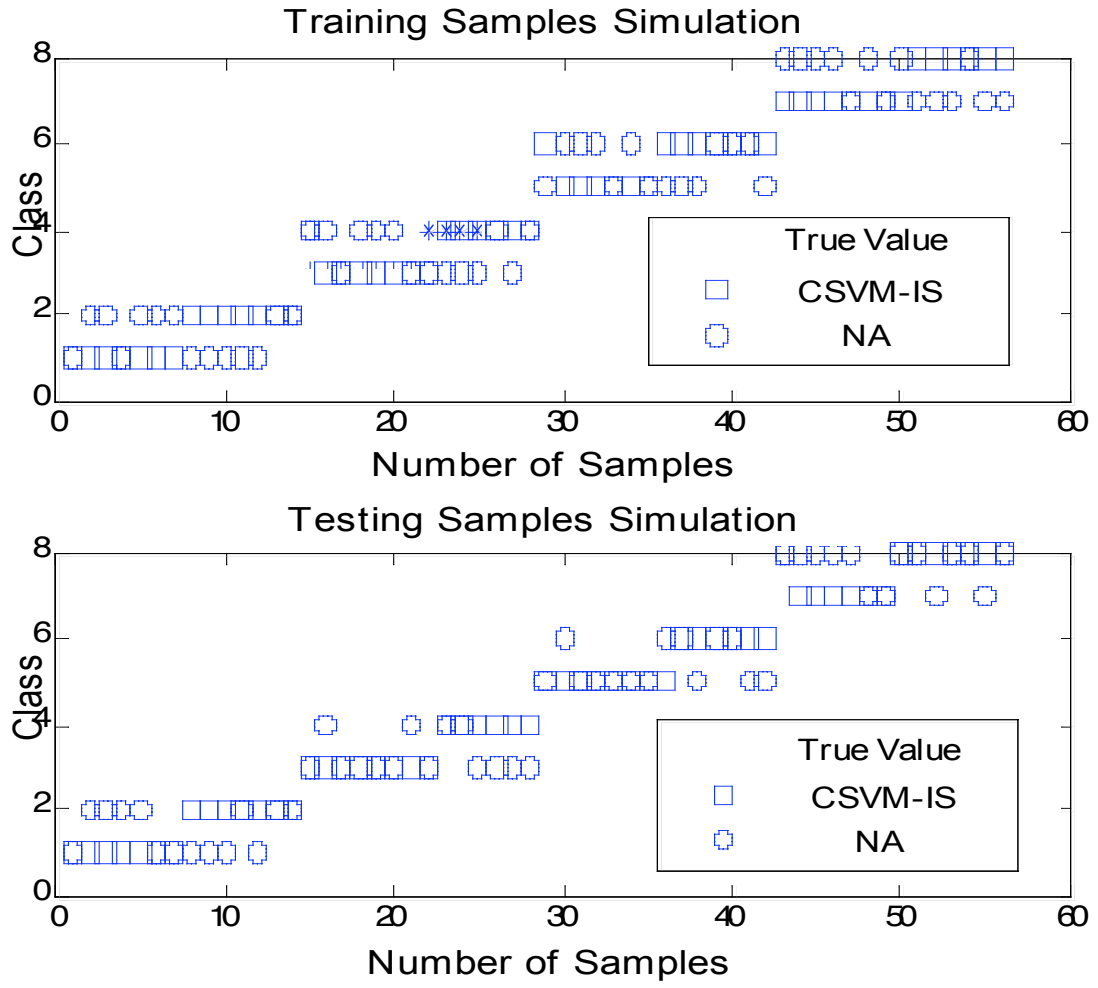

(d)

Fig. (5). Performance simulations for two algorithms of DC1, DC2, DC3 and DC4 respectively in (a), (b), (c), (d). 


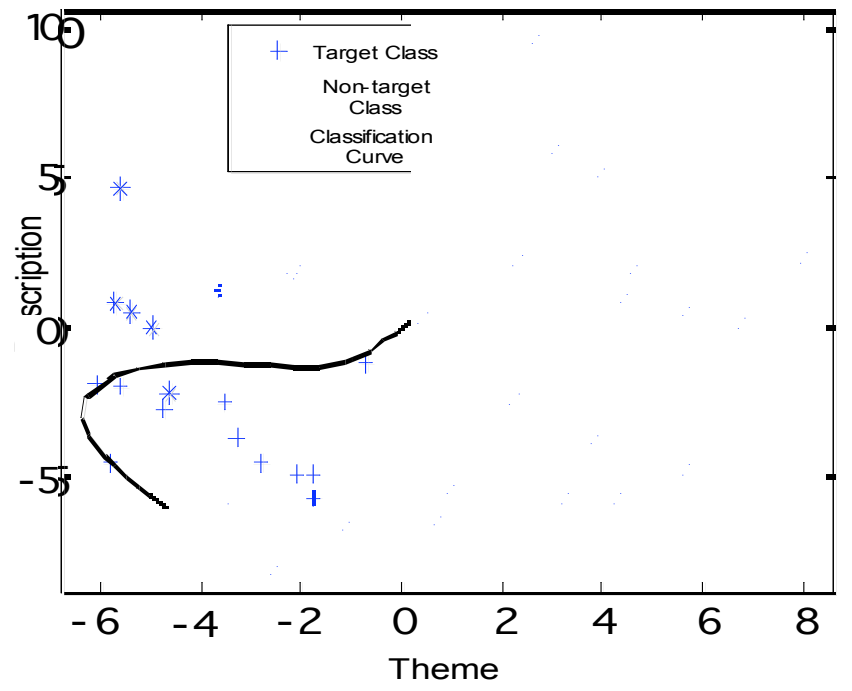

(a) Classification simulation for DC1

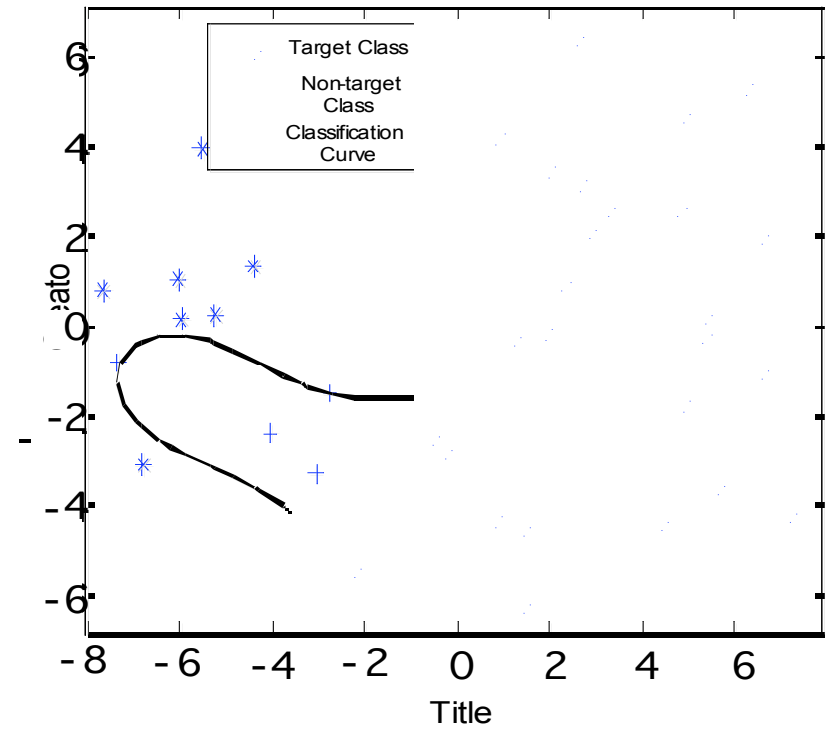

(b) Classification simulation for DC2

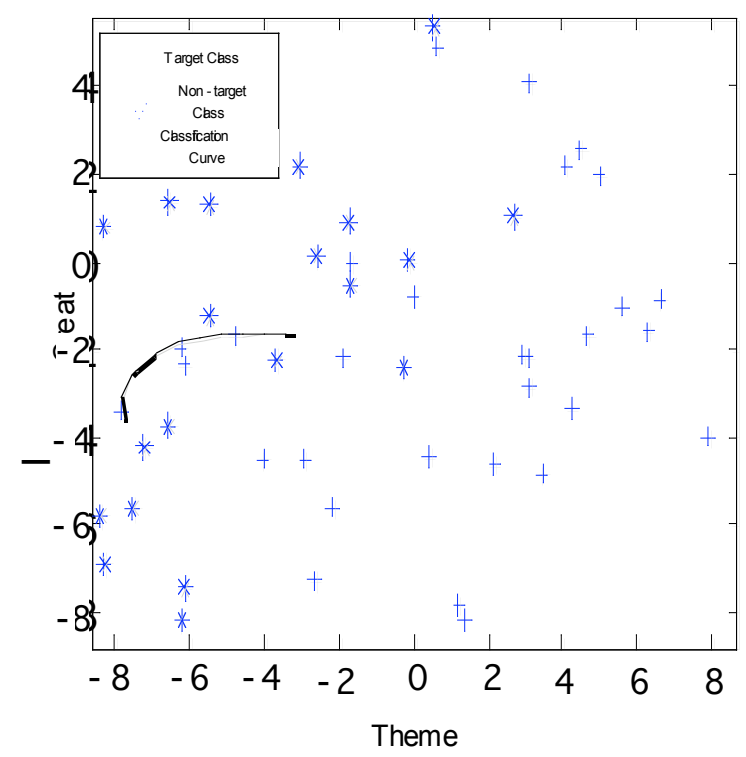

(c) Classification simulation for DC3 


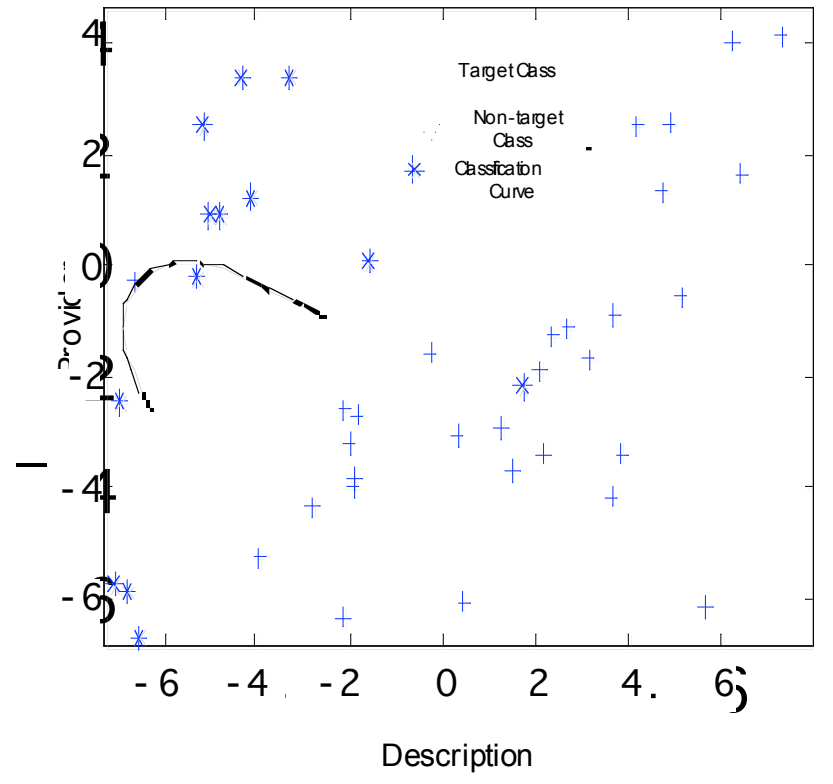

(d) Classification simulation for DC4

Fig. (6). Performance simulations of DC1, DC2, DC3 and DC4 respectively in (a), (b), (c), (d).

axis represents description. In the classification for $60 \mathrm{se}-$ mantic samples of DC1, three non-target samples are assigned to the target class, so accuracy rate of CSVM-IS is about $95 \%$. In (b), the abscissa indicates title, and the vertical axis represents the creator, for DC2, the number of nontarget is three, so the accuracy for semantic classification is about $95 \%$. In (c), abscissa indicates the theme, and the vertical axis represents creator, for DC3 with 60 training samples, the number of non-target misclassification is 4 , therefore the classification accuracy is higher than $90 \%$. In (d), the horizontal axis represents the description, and the vertical axis represents provider, the error number of classification is 3 for DC4. Therefore, all accuracy of CSVM-IS are greater than $90 \%$. Therefore, CSVM-IS can effectively meet user's demands.

Through above simulations, it is known that classification algorithm in this paper has higher classification precision in coping with multi-category image semantic resource and users' needs in retrieval for target class.

\section{CONCLUSIONS}

According to the weak semantic correlation among each image, UCL-I is put forward to express multi-semantic information. And the characteristic values of cloud model can be calculated to determine SCCD. At the same time, CSVMIS is proposed to achieve the automatic semantic classification, and it can effectively extract image semantic features and accomplish target classification. The semantic multiclassification simulation experimental results show that CSVM-IS can effectively learn multiple semantics of image information, and the learning precision is better than NA. And the experimental results of semantic target class show that the classification algorithm can effectively learn the multi-semantic information and has higher classification precision.

\section{CONFLICT OF INTEREST}

The authors confirm that this article content has no conflicts of interest.

\section{ACKNOWLEDGMENTS}

This work was supported by the National Natural Science Foundation of China under NSFC grants 60932005, 60902021 and 61171109.

\section{REFERENCES}

[1] Z. Zhang, S. Chart and L. Chia, "Image classification using tensor representation", in Proceedings of the 15th International Conference on Multimedia, pp. 281-284, 2007.

[2] L. Wu, M. Li, Z. Li, W. Y. Ma and N. Yu, "Visual language modeling for image classification", in Proceedings of the International Workshop on Multimedia Information Retrieval, pp. 115-124, 2007.

[3] B. Subbiah and S. Christopher, "Image classification through integrated K-Means algorithm", International Journal of Computer Science Issues, vol. 9, no.2, pp. 518-524, 2012.

[4] R.A Zneit, A.A Jazar, B. Ayyoub, "Automatic color Images Classification Algorithm”, International Journal of Computer Science Issues, vol.9, no.1, pp. 305-310, 2012.

[5] S.A Nene, S.K Nayar and H. Murase, "Columbia object image library", coil-100, 2008.

[6] W.N. Wang, Y.G. Yu, J.C. Zhang. "A New Svm Based Emotional Classification of Image", Journal of Electronics (China), vol.1, pp. 98-104, 2005.

[7] A.J. Joshi, F. Porikli, N. Papanikolopoulos, "Multi-class active learning for image classification", In 2009 IEEE Conference on Computer Vision and Pattern Recognition, pp. 2372-2379, 2009.

[8] L. Zeng, X. Wu, P. Liu, "Research on SVM and its application of remote sense image classification for regions of interest", Computer Engineering and Applications, vol. 45, no. 6, pp. 243-245, 2009.

[9] H.P. Huang, Y.H. Liu "Fuzzy support vector machines for pattern recognition and data mining", International Journal of Fuzzy Systems, vol. 4, no. 3, pp. 826-835, 2002.

[10] D. Li, H. Meng and X. Shi, "Membership clouds and member cloud generators", Journal of Computer Research and Development, vol. 32, no. 6, pp. 16-21, 1995. 
[11] X. Wang, Z. Shi and S. Wang, "Fuzzy Space Information Processing", Wuhan University Press: Wuhan, 2003.

[12] M.A. Jianguo, L. Xing, Y. Li and Z. Li. "UCL Indexing and Transmission Scheme in Data Broadcasting", Acta Electron Sin, vol. 32, no. 10, pp. 1621-1624, 2004.
[13] L. Xing, J. Ma, Y. Li and Z. Liu. "An information filtering method for chinese web pages based on UCL", Acta Eleciron Simca, vol. 34, no. 10, pp. 1752-1757, 2006

[14] http://www.vision.caltech.edu/Image_Datasets/Caltech101/

[15] B. Liu, Z. Hao and X. Yang, "Nesting algorithm for multiclassification problems", Softw Comput., vol. 11, no.4, pp. 383-389, 2007.

Received: August 13, 2013

(C) Xing et al; ; Licensee Bentham Open.

This is an open access article licensed under the terms of the Creative Commons Attribution Non-Commercial License (http://creativecommons.org/licenses/by-nc/3.0/) which permits unrestricted, non-commercial use, distribution and reproduction in any medium, provided the work is properly cited. 